\title{
Global solution of VLBI observations and assessments
}

\author{
Jinling Li and Guangli Wang \\ Joint Radio Astronomy Research Laboratory, Shanghai Astronomical Observatory, National Astronomical Observatory, \\ Chinese Academy of Sciences, 80 Nandan Road, Shanghai 200030, P. R. China
}

(Received December 31, 1999; Revised May 17, 2000; Accepted May 19, 2000)

\begin{abstract}
Mark III VLBI observations between August 1979 and December 1998 were analyzed to yield the terrestrial and celestial reference frames (TRF and CRF) and the Earth Orientation Parameters (EOP). Comparisons with ITRF96, RSC (WGRF) 95 R01 and EOP (IERS) 97 C04 are performed. For CRF, the three orientation angles are not significant at the level of precision of 0.1 mas. Though no significant values are found for the three deformation parameters, coordinate drifts up to 0.5 mas are identifiable for some sources in the southern hemisphere. The relative rotation angles and their rates of change for TRF are not significant respectively at the precision level of 0.2 mas and $0.1 \mathrm{mas} / \mathrm{yr}$. Detailed comparisons however show that the differences in the velocity field are obvious for the eastern part of Eurasian plate and Australian plate. About EOP series, the systematic differences and the relative drifts are not significant respectively at the level of 0.4 mas and 0.1 mas/yr.
\end{abstract}

\section{Introduction}

Mark III VLBI observations (Ma et al., 1997) between August 1979 and December 1998 are analyzed to yield the terrestrial and celestial reference frames (TRF and CRF) and the Earth Orientation Parameters (EOP). The reduction software system is CALC/SOLVE/GLOBL (Ma et al., 1986). The basic models used to calculate the VLBI delays are generally consistent with the 1996 IERS Conventions (McCarthy, 1996). The terrestrial reference frame is connected to ITRF96 (Boucher et al., 1998) at 1997.0 by applying no-net-horizontal-translation and no-net-rotation constraints to the position adjustments of 12 stations with uniform station weighting for both constraints. The evolution of TRF is connected to NNR-NUVEL1A (DeMets et al., 1994) by applying no-net-horizontal-translation and no-netrotation constraints to the velocity adjustments of five stations with uniform station weighting for both constraints. The celestial reference frame is connected to RSC (WGRF) 95 R01 (ICRF) (Ma et al., 1997) by applying no-net-rotation constraint to the position adjustments of the ICRF defining sources with weighting proportional to the precision of the source positions. The mapping function of Niell (1996) is used for the correction of troposphere delay with cut-off angle at 7 degrees. Clock behavior and wet troposphere effects are modeled as piecewise linear functions. Axis offset and asymmetric atmosphere effects are considered as well. Source positions, station positions and velocities are estimated as global parameters, Earth Orientation Parameters $x, y, U T 1, \delta \psi$ and $\delta \epsilon$ are estimated as arc ones. In total 2,196,201 dual-frequency Mark III group delays from 2,893 full-day sessions are included in the solution. The weighted rms post-fit delay residual is $25.0 \mathrm{ps}$ with reduced $\chi^{2}$ of 1.0. The station coordinates and velocities at J1997.0, the

Copy right (C) The Society of Geomagnetism and Earth, Planetary and Space Sciences (SGEPSS); The Seismological Society of Japan; The Volcanological Society of Japan; The Geodetic Society of Japan; The Japanese Society for Planetary Sciences. source positions at $\mathrm{J} 2000.0$ and the Earth orientation series are designated respectively as SSC (SHAO) 99 R01, RSC (SHAO) 99 R01 and EOP (SHAO) 99 R01. Comparisons with ITRF96, ICRF and EOP (IERS) 97 C04 (IERS, 1998) are performed in the following sections.

\section{Celestial Reference Frame}

Radio source catalogues (RSC) are usually compared by modeling coordinate inconsistencies as the following equation (IERS, 1998)

$$
\begin{aligned}
& A_{1} \tan \delta \cos \alpha+A_{2} \tan \delta \sin \alpha-A_{3}+D_{\alpha}\left(\delta-\delta_{0}\right) \\
& \quad=\alpha_{1}-\alpha_{2} \\
& -A_{1} \sin \alpha+A_{2} \cos \alpha+D_{\delta}\left(\delta-\delta_{0}\right)+B_{\delta}=\delta_{1}-\delta_{2}
\end{aligned}
$$

where $A_{1}, A_{2}$ and $A_{3}$ are three orientation angles, $D_{\alpha}, D_{\delta}$ and $B_{\delta}$ are three deformation parameters representing respectively drifts in right ascension and in declination as functions of declination, and a bias in declination (for $\left.\delta_{0}=0\right)$. $\left(\alpha_{1}, \delta_{1}\right)$ and $\left(\alpha_{2}, \delta_{2}\right)$ are coordinates of a source, and $(\alpha, \delta)$ can be either $\left(\alpha_{1}, \delta_{1}\right)$ or $\left(\alpha_{2}, \delta_{2}\right)$ which leaves no sensible difference in solution. The relative orientation and deformation parameters between our solution and ICRF are evaluated by a weighted least square fit of the above equation. Results are listed in Table 1, in which corresponding values for two other RSCs from IERS (1998) are also listed for comparison. These RSCs had the smallest and largest orientation differences respectively relative to ICRF of the IERS comparisons made. The results are based on all the common sources for the last column and based on only defining sources for other columns.

All the VLBI frames in Table 1 have been tied to ICRF by imposing constraints to the adjustments of coordinates of some selected sources. For instance, the orientation of RSC (GIUB) 98 R01 is defined by fixing the ICRF right ascension of $2145+67$ and $0528+134$ and the declination of $2145+$ 
Table 1. Relative orientation and deformation parameters in the sense from individual VLBI frame to ICRF. $r_{\alpha}$ and $r_{\delta}$ are the weighted rms residuals in $\alpha \cos \delta$ and $\delta$ respective. $D_{\alpha}$ and $D_{\delta}$ are in mas/degree, others are in mas.

\begin{tabular}{ccccc}
\hline & RSC (GIUB) 98R01 & RSC (USNO) 98R06 & RSC (SHAO) 99R01 & RSC (SHAO) 99R01 \\
$\mathrm{N}$ & 206 (defining) & 211 (defining) & 212 (defining) & 606 (all the sources) \\
\hline$A_{1}$ & $0.552 \pm 0.017$ & $0.020 \pm 0.017$ & $0.013 \pm 0.015$ & $0.015 \pm 0.010$ \\
$A_{2}$ & $-0.431 \pm 0.017$ & $0.033 \pm 0.017$ & $-0.018 \pm 0.015$ & $-0.024 \pm 0.010$ \\
$A_{3}$ & $0.011 \pm 0.022$ & $-0.031 \pm 0.022$ & $-0.064 \pm 0.019$ & $-0.014 \pm 0.011$ \\
$D_{\alpha}$ & $-0.002 \pm 0.001$ & $-0.002 \pm 0.001$ & $-0.002 \pm 0.001$ & $-0.000 \pm 0.001$ \\
$D_{\delta}$ & $-0.001 \pm 0.000$ & $0.000 \pm 0.000$ & $0.000 \pm 0.000$ & $0.000 \pm 0.001$ \\
$B_{\delta}$ & $0.019 \pm 0.017$ & $0.058 \pm 0.018$ & $-0.027 \pm 0.015$ & $-0.021 \pm 0.010$ \\
$r_{\alpha}$ & \pm 0.19 & \pm 0.17 & \pm 0.16 & \pm 0.19 \\
$r_{\delta}$ & \pm 0.22 & \pm 0.25 & \pm 0.20 & \pm 0.21 \\
\hline
\end{tabular}

Table 2. Transformation parameters at J1997.0 from our solution to ITRF96.

\begin{tabular}{lr|lr}
\hline$T_{1}(\mathrm{~cm})$ & $0.354 \pm 0.212$ & $\dot{T}_{1}(\mathrm{~cm} / \mathrm{yr})$ & $0.020 \pm 0.038$ \\
$T_{2}(\mathrm{~cm})$ & $-0.012 \pm 0.211$ & $\dot{T}_{2}(\mathrm{~cm} / \mathrm{yr})$ & $-0.218 \pm 0.039$ \\
$T_{3}(\mathrm{~cm})$ & $-0.087 \pm 0.210$ & $\dot{T}_{3}(\mathrm{~cm} / \mathrm{yr})$ & $-0.110 \pm 0.036$ \\
$D\left(10^{-8}\right)$ & $0.072 \pm 0.031$ & $\dot{D}\left(10^{-8} / \mathrm{yr}\right)$ & $-0.002 \pm 0.005$ \\
$R_{1}(\mathrm{mas})$ & $0.093 \pm 0.084$ & $\dot{R}_{1}(\mathrm{mas} / \mathrm{yr})$ & $0.028 \pm 0.016$ \\
$R_{2}(\mathrm{mas})$ & $-0.194 \pm 0.079$ & $\dot{R}_{2}(\mathrm{mas} / \mathrm{yr})$ & $-0.038 \pm 0.014$ \\
$R_{3}(\mathrm{mas})$ & $0.039 \pm 0.070$ & $\dot{R}_{3}(\mathrm{mas} / \mathrm{yr})$ & $0.107 \pm 0.011$ \\
\hline
\end{tabular}

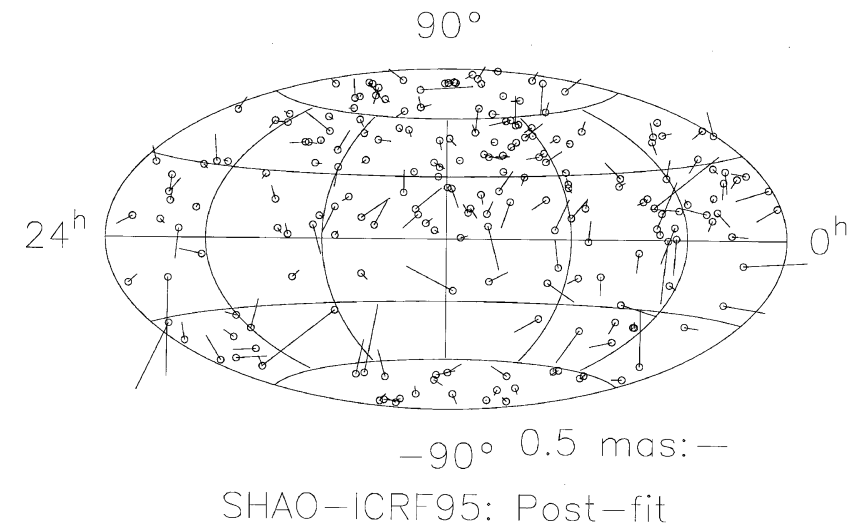

Fig. 1. Residual position differences of defining sources from Eq. (1).

067. While RSC (USNO) 98 R01 is tied directly to ICRF in a minimum no net rotation and translation sense (IERS, 1998). The fitted values of the relative orientation parameters are less than 0.6 mas, and for our solution they are less than 0.1 mas. The adjusted values of the relative deformation parameters are also not significant at the level of precision of 0.1 mas. For the last two columns in Table 1, there exist differences between the adjusted values of parameters by comparing only defining sources with those by comparing all the common sources. However, the corresponding values of parameters are with the same sign and the differences between them are not very significant.

Figure 1 shows the residual differences in positions of the defining sources from Eq. (1). About three dozens of defining sources have position differences larger than 0.5 mas, which is slightly significant compared with the mean uncertainty ( 0.4 mas) of the differences. Most of these sources are in the southern hemisphere. However, the large differences in position are not reflected by the adjusted values of deformation parameters (refer to Table 1).

\section{Terrestrial Reference Frame}

By comparing our solution with ITRF96 at J1997.0 as shown in Table 2, the orientation angles and their rates of change are not significant respectively at 0.2 mas and 0.1 mas/yr. However, since the distribution of stations is mostly condensed in Europe and North America, small values in Table 2 imply good consistency between the two solutions within only limited areas. As shown in Fig. 2, the differences in the velocity field are obvious and slightly significant comparing with their formal uncertainties for the eastern part of Eurasian plate and for Australian plate. Therefore a more even distribution of stations will be good to further improving the terrestrial reference frame realized by VLBI technique. 


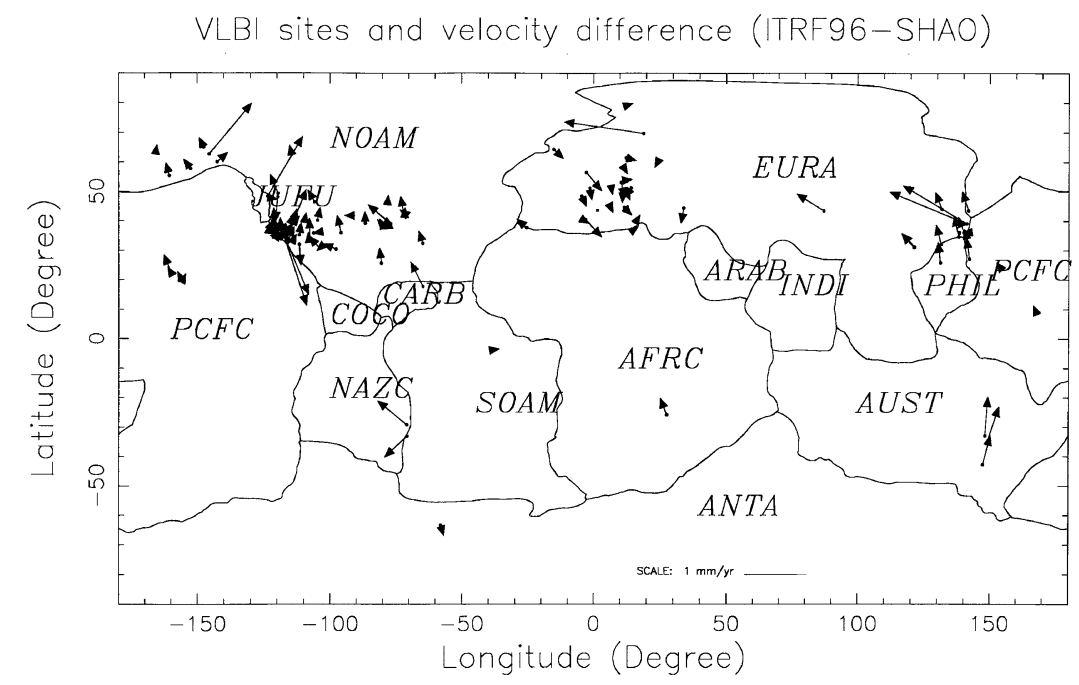

Fig. 2. The difference between our velocity field and ITRF96 at J1997.0 after transformation.

Table 3. The biases and drifts of our solution compared with EOP (IERS) $97 \mathrm{C} 04$.

\begin{tabular}{crr}
\hline & \multicolumn{1}{c}{$a(\mathrm{mas})$} & \multicolumn{1}{c}{$b(\mathrm{mas} / \mathrm{yr})$} \\
\hline$x$ & $0.359 \pm 0.011$ & $0.051 \pm 0.002$ \\
$U T 1-U T C$ & $-0.256 \pm 0.010$ & $-0.007 \pm 0.002$ \\
$\delta \phi$ & $-0.086 \pm 0.014$ & $-0.012 \pm 0.002$ \\
$\delta \epsilon$ & $-0.026 \pm 0.006$ & $-0.001 \pm 0.001$ \\
\hline
\end{tabular}

Table 4. Consistency of IERS EOP series with ITRF and ICRF at J2000.0.

$$
\begin{array}{lc}
(\Delta x)-\left(R_{2}\right) & 0.103 \pm 0.121 \text { (mas) } \\
(\Delta y)-\left(R_{1}\right) & -0.008 \pm 0.128 \text { (mas) } \\
(f \times \Delta U T 1)-\left(-R_{3}+A_{3}\right) & -0.242 \pm 0.108 \text { (mas) } \\
(\Delta d \psi)-\left(A_{2} / \sin \epsilon\right) & -0.131 \pm 0.040 \text { (mas) } \\
(\Delta d \epsilon)-\left(-A_{1}\right) & -0.039 \pm 0.016 \text { (mas) } \\
(\Delta \dot{x})-\left(\dot{R}_{2}\right) & 0.009 \pm 0.015 \text { (mas/yr) } \\
(\Delta \dot{y})-\left(\dot{R}_{1}\right) & 0.015 \pm 0.016(\mathrm{mas} / \mathrm{yr}) \\
(f \times \Delta U T 1)-\left(-\dot{R}_{3}\right) & 0.014 \pm 0.011(\mathrm{mas} / \mathrm{yr})
\end{array}
$$

\section{Earth Orientation}

The systematic differences of our solution compared with EOP (IERS) 97 C04 are shown in Table 3. The biases and the relative drifts are not significant respectively at the precision level of 0.4 mas and $0.1 \mathrm{mas} / \mathrm{yr}$.

From the above comparisons some consistency judgements for the IERS EOP series with ITRF and ICRF can be deduced (IERS, 1998). As shown in Table 4, at J2000.0 the closures are not significantly different from zero at the level of precision of 0.2 mas. For the time evolutions of the IERS EOP series relative to ITRF, they are not significantly different from zero at $0.02 \mathrm{mas} / \mathrm{yr}$.

\section{Concluding Remarks}

Systematic differences in our solutions of CRF, TRF and EOP series from global analysis of VLBI observations are not obvious compared with the IERS solutions. Detail comparisons show that some defining sources in the southern hemisphere have position differences large than 0.5 mas in our solution and ICRF, that a more even distribution of stations are required to further improving the terrestrial reference frame realized by VLBI technique. The closures of IERS EOP series with ITRF and ICRF are about 0.2 mas. The corresponding time evolutions are about $0.02 \mathrm{mas} / \mathrm{yr}$.

Acknowledgments. This work is partly supported by the Chinese National Major Basic Research Project No. 970231003, Major Project of National Natural Scientific Foundation Committee No. 19833030, Major Project of CAS No. KJ951-1-304 and National Major Basic Research Project No. 95-13-03-02.

\section{References}

Boucher, C., Z. Altamimi, and P. Sillard, Results and analysis of the ITRF96, in IERS Technical Note 24, pp. 3-15, Observatoire de Paris, 1998.

DeMets, C., R. G. Gordon, D. F. Argus, and S. Stein, Effect of recent revisions to the geomagnetic reversal time scale on estimates of current plate motions, Geophys. Res. Lett., 21, 2191-2194, 1994.

IERS, 1997 IERS Annual Report, pp. II-35-II-53, Observatoire de Paris, 1998.

Ma, C., E. F. Arias, T. M. Eubanks, A. L. Fey, A. M. Gontier, C. S. Jacobs, O. J. Sovers, B. A. Archinal, and P. Charlot, The international celestial reference frame realized by VLBI, in IERS Technical Note 23, edited by C. Ma and M. Feissel, pp. II-3-II-44, Observatorie de paris, 1997.

Ma, C., T. A. Clark, J. W. Ryan, T. A. Herring, I. I. Shapiro, B. E. Corey, H. F. Hinteregger, A. E. Rogers, A. R. Whitney, C. A. Knight, G. L. Lundqvist, D. B. Shaffer, N. R. Vandenberg, J. C. Pigg, B. R. Schupler, and B. O. Ronnang, Radio-source positions from VLBI, Astron. J., 92, 1020-1029, 1986

McCarthy, D. D., IERS Convensions (1996), in IERS Technical Note 21, pp. 4-94, Observatorie de Paris, 1996.

Niell, A. E., Global mapping functions for the atmospheric delay of radio wavelengths, J. Geophys. Res., 101, 3227-3246, 1996.

J. L. Li (e-mail: j11@center.shao.ac.cn) and G. L. Wang (e-mail: wgl@ center.shao.ac.cn) 